\title{
Intravenous immunoglobulin for neuromuscular disease: costs, benefits and reimbursement
}

\author{
John W Griffin and Richard AC Hughes
}

Nelson Algren entitled his 1949 novel on intravenous drug dependency "The Man with the Golden Arm”. Sixty years later, 'golden arms' could be fittingly used to describe those patients who require, at daunting cost, regular infusions of intravenous immunoglobulin (IVIg). Access to IVIg is at present by no means uniform. Development of a fair and rational system for allocation of IVIg will be one bellwether of the success of health policy reforms.

A European Federation of Neurological Societies (EFNS) task force has recently endorsed, with its highest level of support, long-standing recommendations for the use of IVIg in severe Guillain-Barré syndrome and for acute exacerbations of myasthenia gravis (Elovaara I et al. [2008] Eur J Neurol 15: 893908). Importantly, this group has also endorsed recent recommendations for the use of IVIg in chronic inflammatory demyelinating polyradiculoneuropathy (CIDP) and multifocal motor neuropathy. These recommendations, from the joint task forces of the EFNS and Peripheral Nerve Society, have been made on the basis of results from randomized, controlled trials summarized in Cochrane systematic reviews. These reviews are regularly updated, an intention that has yet to be realized for the guidelines.

The disorders for which IVIg has been approved do not match on the different sides of the Atlantic. The ICE trial (Hughes RA et al. [2008] Lancet Neurol 7: 136-144) strengthened the evidence for use of IVIg in CIDP and persuaded the FDA to license an IVIg product for this disease. CIDP is the first neuromuscular disorder for which an IVIg product has been licensed in the US. By contrast, the European Medicines Agency (EMEA) has not licensed any IVIg product for use in CIDP. Conversely, in Guillain-Barré syndrome, IVIg is approved by the EMEA but not by the FDA. These mismatches arise from limitations of evidence, different grading of evidence by different organizations, low prevalence of the disorders and the resulting
Development

of a fair and

rational system

for allocation of

IVlg will be one

bellwether of

the success

of health policy

reforms

JW Griffin is the

Editor-in-Chief

of Nature Clinical

Practice Neurology.

RAC Hughes is

Emeritus Professor

of Neurology at

King's College London

and Visiting Professor

of Neurology at

University College

London, London, UK

Competing interests

JW Griffin has declared

an association with the

following company:

Biogen-Idec. RAC Hughes

has declared associations

with the following

companies: Baxter,

Octapharma, Talecris and

ZLB Behring. See the article

online for full details of the

relationships.

www.nature.com/clinicalpractice doi:10.1038/ncpneuro1046 small scale of trials, and inertia of both licensing systems. In the US, some patients who demonstrate repeated and dramatic response to IVIg experience difficulties with access to the agent, while anecdotal evidence suggests that others have overused it. This inconsistency reflects the fragmented US health-care system and is arguably amplified by the higher reimbursement for IVlg than for alternatives such as corticosteroids or immunosuppressives. Of note, the English National Health Service has now laid down guidelines for all IVIg usage and put in place a monitoring system to regulate use of this agent and a database to collect information about actual usage.

Few studies have investigated the costeffectiveness of IVIg in neuromuscular disease. In one short-term study (McCrone P et al. [2003] Eur J Neurol 10: 687-694), a likelihood of IVIg being more cost-effective than prednisolone in CIDP occurred only if one quality-of-life-year was valued at over $€ 250,000$. The long-term adverse effects of prednisolone will undoubtedly affect this calculation, but the cost of IVIg is undeniably high and is likely to remain so.

In that bright future of rational allocation of health-care expenses to which all nations aspire, investment will be needed in adequate studies to determine efficacy and cost-effectiveness. Some patients do not fit within strict diagnostic categories that were established to ensure homogeneous research groups. Furthermore, even among correctly diagnosed patients, individual responses can vary-not every patient with CIDP, for instance, should receive long-term IVlg therapy. We need mechanisms to 'try out' and document the extent of benefit in individual patients, coupled with a protocol for repeated assessments to determine whether benefit is continued. Finally, in deciding the appropriateness of IVIg for individuals in terms of cost and benefit, we will need protocols that factor in the long-term costs of cheaper but more-toxic alternatives to IVIg, such as corticosteroids. 